\title{
Strates
}

STRATES Matériaux pour la recherche en sciences sociales

8 | 1995

La question de l'environnement: recherches parallèles en Espagne et en France

\section{Biogéographie d'un espace forestier méditerranéen espagnol}

Vicente Jurado Doña

\section{(2) OpenEdition \\ Journals}

Édition électronique

URL : http://journals.openedition.org/strates/1050

DOI : $10.4000 /$ strates. 1050

ISSN : $1777-5442$

Éditeur

Laboratoire Ladyss

Édition imprimée

Date de publication : 30 juin 1995

ISSN : 0768-8067

Référence électronique

Vicente Jurado Doña, « Biogéographie d'un espace forestier méditerranéen espagnol », Strates [En ligne], 8 | 1995, mis en ligne le 20 décembre 2005, consulté le 08 septembre 2020. URL : http:// journals.openedition.org/strates/1050 ; DOI : https://doi.org/10.4000/strates.1050

Ce document a été généré automatiquement le 8 septembre 2020

Tous droits réservés 


\title{
Biogéographie d'un espace forestier méditerranéen espagnol
}

\author{
Vicente Jurado Doña
}

\section{NOTE DE L'ÉDITEUR}

Traduit de l'espagnol par Marie-Laure Drouet et Michèle Petit. Relu par Marie-Claude Guerrini.

1 L'importance des ressources forestières dans les régions de climat méditerranéen est bien connue, et elle a été étudiée par de nombreux auteurs, tout comme l'influence que l'activité humaine a eu sur ces ressources depuis plusieurs millénaires (Vernet, 1973 ; Pons, 1985 ;Thirgood, 1981 ; Stevenson, 1992).

2 En Andalousie, située au sud de la péninsule ibérique, un peu plus de la moitié de la superficie (4,6 millions d'hectares sur 8,7$)$ est considérée comme étant à vocation forestière, c'est-à-dire constituée de terrains plantés $(51,3 \%)$ ou de matorrales $^{1}(48,7 \%)$ qui sont impropres à l'agriculture (Junta de Andalucia, 1992). Au-delà d'une première impression positive quant à l'étendue des espaces arborés, nous devons constater qu'une grande partie d'entre eux ne peuvent être considérés comme d'authentiques forêts, au sens écologique du terme - où les diverses strates (arborée, arbustive, herbacée et lianoïde) maintiennent d'étroites relations entre elles et avec les facteurs géomorphologiques, climatiques et édaphiques. Dans les dernières décennies, en Andalousie tout comme dans d'autres régions de l'État espagnol, la forêt a souffert d'une profonde et intense transformation qui a entraîné la disparition d'immenses surfaces arborées originelles et l'implantation d'espèces à croissance rapide, provoquant des changements significatifs, tant dans le paysage que dans la dynamique naturelle des forêts (Castroviejo,1985; Groome, 1990).

3 La forêt originelle est fondamentalement constituée par des espèces du genre Quercus (famille des Fagaceae) qui couvriraient à peu près 1,2 millions d'hectares, et dont la plus grande partie est constituée de chênaies et de bois de chênes-lièges pâturés avec un 
taux de recouvrement inférieur à $25 \%$, qui représentent la formation végétale la plus étendue et la plus connue.

4 A côté des chênes (Quercus rotundifolia) et des chênes-lièges (Quercus suber) d'autres formations végétales, bien que moins étendues, remplissent diverses fonctions écologiques et paysagères. C'est le cas des forêts de pinsapos (Abies pinsapo) des hauteurs de Malaga et Cadix, découvertes pour la science à la fin du XIX ${ }^{\mathrm{e}}$ siècle par le botaniste suisse Boissier, qui sont un authentique joyau botanique pour l'Andalousie et l'Europe. Ces forêts, actuellement protégées par l'Agence de l'environnement d'Andalousie, sont apparentées, d'un point de vue taxinomique, aux forêts des montagnes marocaines, et elles montrent une nette préférence pour les adrets et pour une pluviométrie élevée (entre 2000 et $3000 \mathrm{~mm}$ par an).

5 Il faut aussi relever l'existence de bois d'oliviers sauvages (Olea europaea var. sylvestris) sur des substrats de vertisols, sols ayant une forte concentration argileuse, typiques de certaines zones comme la région de Cadix. Les oliviers sauvages y sont associés au lentisque (Pistacia lentiscus) et à d'autres espèces arbustives, formant ainsi un paysage typique du piémont.

6 Les pinèdes (on compte jusqu'à six espèces différentes du genre Pinus) couvrent $32 \% \mathrm{de}$ la superficie forestière arborée andalouse : c'est dire l'importance de leur étendue. Les pins ont acquis un poids important dans les repeuplements forestiers effectués au cours des dernières décennies par l'ICONA, l'organisme compétent pour les questions forestières au niveau national, mais leur présence antérieure est connue, dans les franges littorales des provinces de Huelva et Cadix, au moins depuis le premier tiers du XVIII ${ }^{e}$ siècle (Granados, 1987). Dans cet espace côtier, principalement dans la zone de Huelva, a dû exister une forêt de genévriers (Juniperus oxycedrus sous-espèce macrocarpa) et de genévriers de Phénicie (Juniperus phoenicea sous-espèce turbinata) qui a presque totalement disparu du fait de l'action destructrice de l'homme, seuls quelques spécimens subsistant aujourd'hui.

7 A côté de cette représentation variée de formations forestières méditerranéennes, nous trouvons aussi des formations de matorrales qui jouent un rôle écologique important dans les différents étages bioclimatiques de la région - formations bien associées dans leur étape juvénile, pionnières de la succession écologique, ou constituant le matorral noble dans ses étapes climacique ou avancée.

Origine des forêts méditerranéennes

8 La forêt sclérophylle méditerranéenne est la formation végétale la plus importante du bassin méditerranéen. Ses caractéristiques actuelles reflètent son évolution passée et les différentes adaptations qu'elle a connues en relation avec les changements survenus dans le milieu.

9 La végétation à caractère méditerranéen existe depuis au moins cinq millions d'années (début du Pliocène), mais sa présence était alors peu importante puisque les principales forêts étaient constituées par des espèces à feuilles larges et lustrées (Laurisilva), adaptées à un climat plus humide et plus chaud qu'actuellement, bien que comportant déjà une saison sèche (Suc, 1985). Les changements survenus avec l'arrivée des oscillations glaciaires sur la végétation furent radicaux. Le maximum de la dernière glaciation - Würm - se produisit il y a environ 20000 ans, agissant de manière destructrice sur l'Europe moyenne. 

$\mathrm{du}$ Quaternaire, et elles ont servi de refuge pour une partie de la flore du Tertiaire, dont l'extinction fut évitée. Certaines enclaves d'Andalousie ont ainsi permis la sauvegarde de nombreuses espèces alpines et boréales (Blanca, 1993). On admet généralement que, durant une bonne partie de l'histoire de l'humanité, les groupes de chasseurs-cueilleurs agissaient sur la forêt, mais que leur comportement permettait l'homéostasie et la préservation du système forestier (Gonzalez, 1989). Pendant le Néolithique, les activités pastorales et agricoles eurent une incidence notoire et intense sur la forêt. Les sociétés préromaines, du début du Néolithique jusqu'à l'Age du fer, semblent avoir été celles qui entamèrent un processus de déforestation durable dans plusieurs régions. Avec l'intensification des pratiques agropastorales au Néolithique, coïncidant avec certaines valeurs de pluviométrie plus élevées pendant la période atlantique (il y a 8000 ans), les forêts de Quercus qui prédominaient nettement dans l'espace forestier de la péninsule ibérique, en coexistence avec d'autres formations - principalement des pinèdes, des genévriers, des sabines et des oliviers sauvages (Costa, 1990) -, furent transformées.

Parc naturel de los Alcornocales

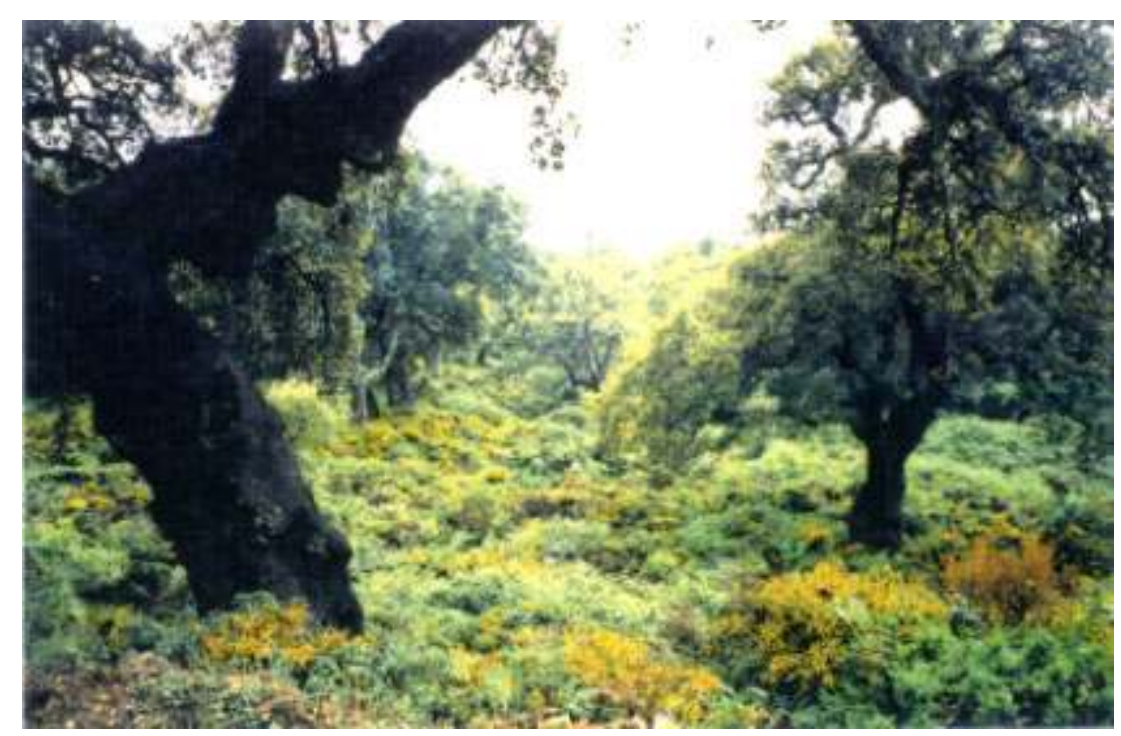

Dehesa à chênes-lièges 
Parc naturel de los Alcornocales

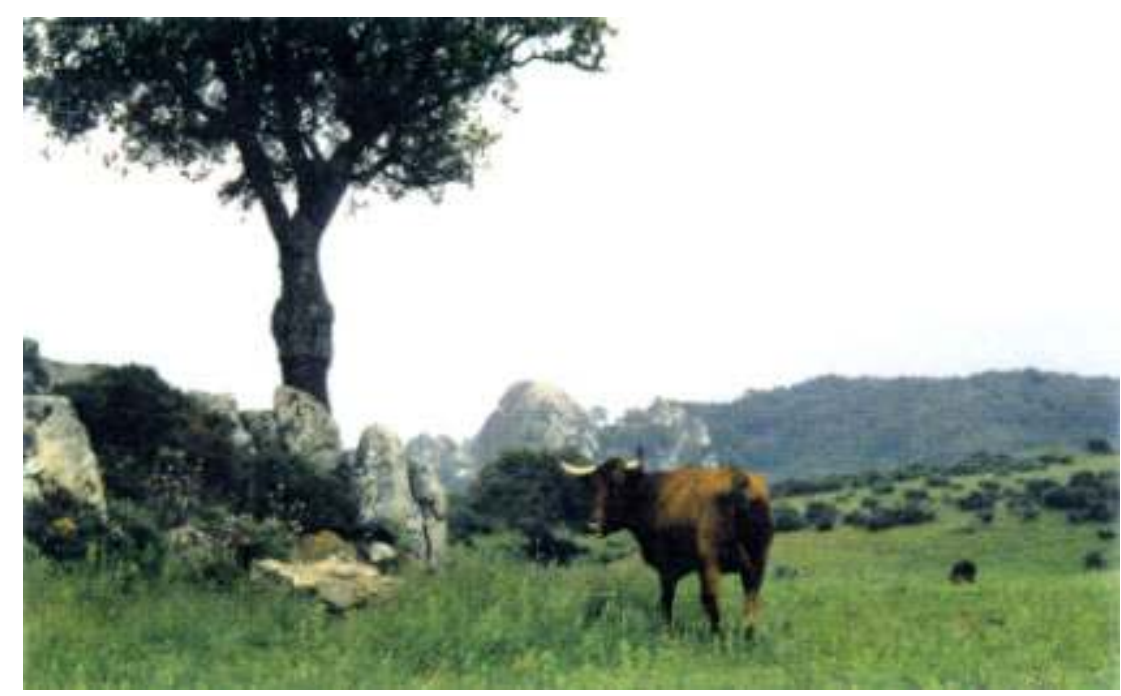

Vache de la race retinta dans une clairière pastorale de la même dehesa

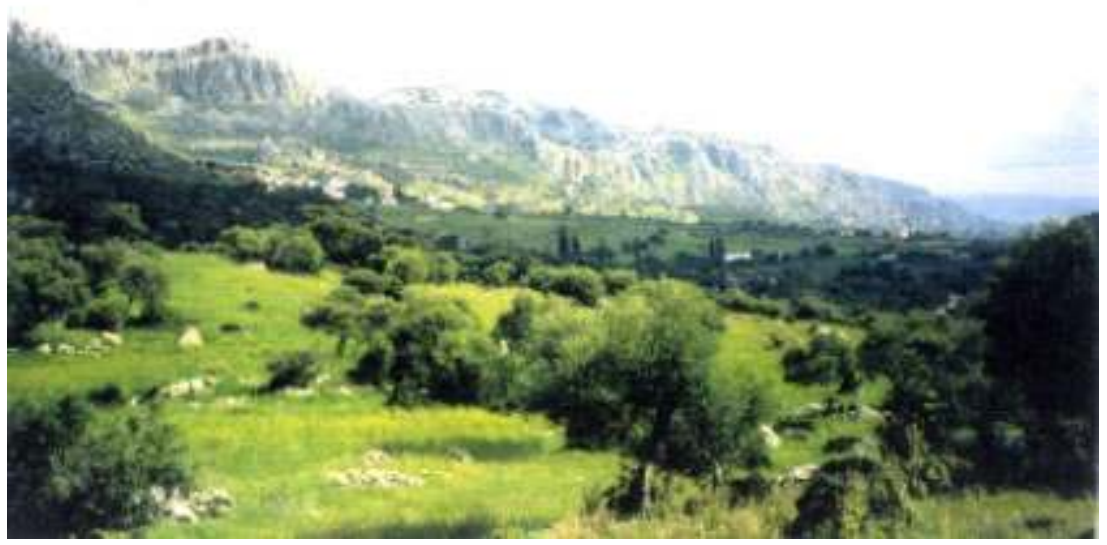

Sierra de Cadix

12 Récemment, pour les forêts de pins proches du Parc National de Doñana (Huelva, Séville), des chercheurs britanniques ont reconstitué le milieu forestier des dernières six mille années, grâce à des analyses de pollens recueillis dans des échantillons de tourbes et de sédiments (Stevenson, 1992). Selon ces auteurs, des changements significatifs sont intervenus dans la composition de la végétation entre 4000 avant J.-C. et 1900 de notre ère, qui ne s'accordent pas à la succession naturelle de la végétation méditerranéenne de cette région, et que l'on interprète comme résultant des actions délibérées des communautés humaines qui vivaient à proximité. Le sous-bois de chênes (Quercus spp.) a été réduit par l'action des pâturages et des feux, alors que les arbres étaient conservés pour le bois de chauffe et les fruits de la forêt. Depuis des millénaires

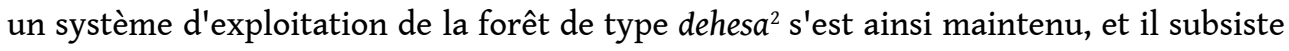
encore de nos jours dans de larges zones d'Andalousie, mais aussi dans d'autres régions espagnoles et portugaises (Marañon, 1988). L'importance écologique actuelle de la dehesa trouve sa source dans sa configuration et sa permanence comme écosystème en 
authentique équilibre, que l'homme a su modeler et modifier, créant autour de ce système d'exploitation toute une culture savante, un ensemble de connaissances du milieu naturel, incluant arbres et bétail (Jurado, 1992).

L'élevage et le feu, facteurs altérant la forêt

Pendant le Moyen Age, l'impact sur la forêt a dû rester circonscrit à des enclaves localisées. Les communautés du Haut Moyen Age étaient de simples hameaux au milieu de la forêt qui était l'élément dominant du paysage, malgré l'avancée des défrichements et des semailles de céréales (blé, avoine, seigle, orge) et de légumes (pois et fèves). L'extension des terres de labour, bien que limitée par les structures propres au système féodal, fut suffisante pour amorcer la disparition de certaines espèces végétales et animales (Hernandez, 1985).

14 A la fin du XIII ${ }^{e}$ siècle, le roi Alphonse X le Sage légalisa la " Mesta $»^{3}$, une institution qui semble avoir joué un rôle important, voire décisif, dans la transformation des paysages méditerranéens ibériques - même si certains auteurs attribuent la déforestation à la lutte séculaire entre royaumes chrétiens du nord de la péninsule et arabes du sud, à l'exploitation minière, à la consommation d'arbres pour l'Armada espagnole lors de l'expansion de l'Empire, et à l'intense et continuelle activité agricole (Parra, 1988). Quoi qu'il en soit, les bergers transhumants jouirent de nombreux privilèges et il y eut beaucoup d'atteintes aux montagnes et aux friches des villages situés sur les grandes voies empruntées par les troupeaux de brebis entre les pâturages d'été et les quartiers d'hiver. L'exportation des laines de brebis mérinos et la consolidation d'une puissante industrie textile atteignirent leur apogée sous le règne des Rois catholiques (1474-1516).

La Mesta procura à la couronne de Castille des rentrées d'argent considérables grâce au commerce des laines des ovins transhumants et aux péages obligatoires qui devaient être acquittés pour les troupeaux à certains passages ou ponts. L'Honorable Conseil de la Mesta devint l'entreprise la plus importante du pays et assura la prépondérance de la Castille sur le reste des royaumes péninsulaires. Il n'y eut rien de comparable dans le bas Moyen Age et au cours des Temps modernes, et les laines espagnoles envahirent les marchés du monde (Saenz, 1986). Toutefois, il faut signaler que seule une petite partie du cheptel ovin castillan transhumait. Pendant le XVI ${ }^{e}$ siècle, celui-ci a parfois dépassé les trois millions de têtes, et le commerce intérieur en Castille se concentrait dans plusieurs centres urbains. Du fait de la politique nationale des Rois catholiques, l'industrie lainière espagnole cessa d'être une activité irrégulière et donna lieu à de vastes échanges à l'échelle nationale, reliant entre eux les différents centres commerciaux (Klein, 1936). 
EVOLUTION DES INCENDIES FORESTIERS EN ANDALOUSIE (1970 - 1992)

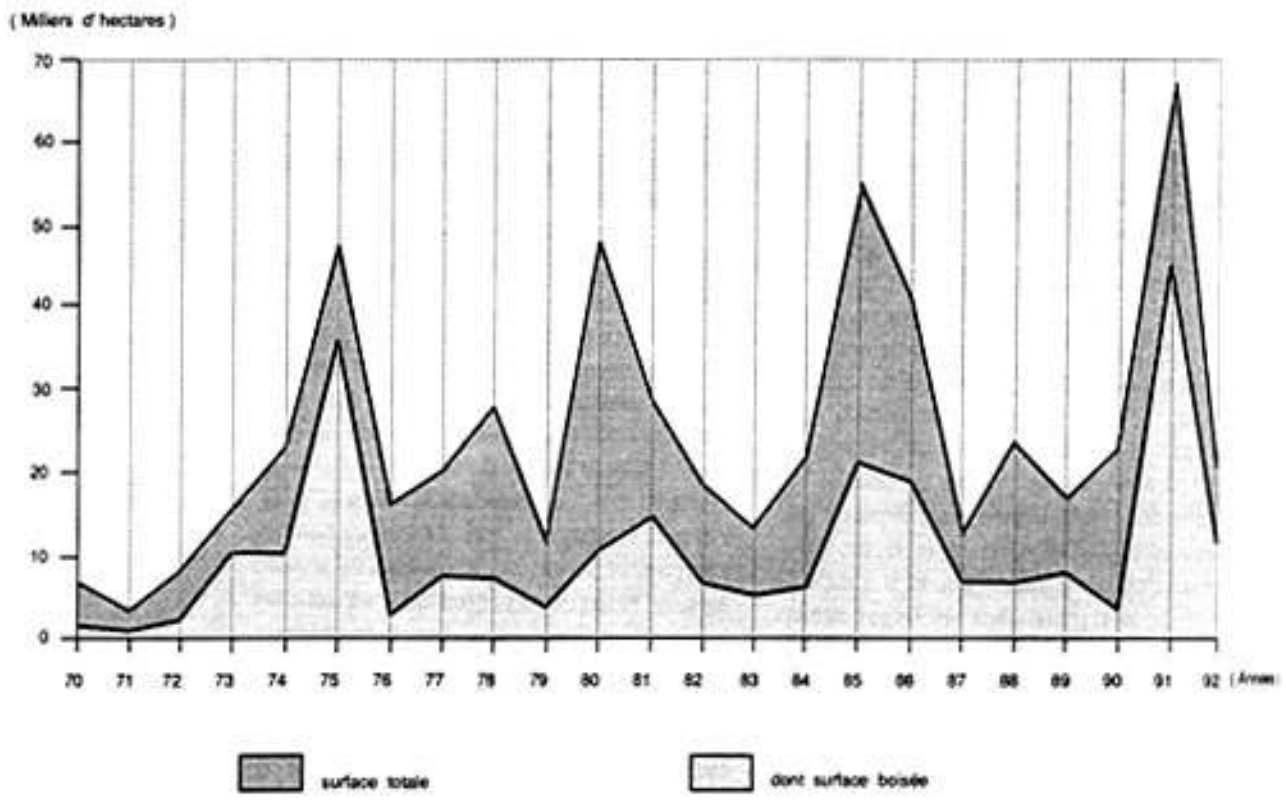

Tant les Rois catholiques que leurs successeurs prirent de nombreuses dispositions qui tendaient à encourager la transhumance, mais qui par là-même nuisaient à l'agriculture et aux forêts (Bauer, 1980). Le réseau de chemins pour les troupeaux était parvenu à couvrir une longueur de 125000 kilomètres et une surface de 500000 hectares (Perez,1991).

Quand l'affaissement du commerce de la laine s'amorça, tant à l'extérieur qu'à l'intérieur, il produisit inévitablement l'affaiblissement simultané de la Mesta, dont les activités commerciales déclinèrent rapidement en même temps que son influence et sa puissance (Klein, op. cit.). Divers facteurs contribuèrent à la chute de cette institution, qui détint durant des siècles le monopole mondial du commerce de la laine notamment le déclin de l'élevage et la profonde transformation des modes d'élevage traditionnel. Le coup de grâce fut peut-être la première exportation à grande échelle des brebis mérinos. L'installation florissante de troupeaux en Suède, en 1720, puis en Saxe et à Rambouillet, rendit inévitable le déclin puis la mort de la Mesta, dont les méthodes étaient surannées, tant pour le commerce d'exportation que pour le pâturage (Klein, op. cit.)

18 A son apogée, l'élevage a eu des conséquences non seulement morphologiques (déforestation massive), mais aussi structurelles (luttes pour les communaux qui prenaient de la valeur et tendaient à être privatisés). Elles s'ajoutèrent aux changements imputables à la Marine de guerre qui, au faîte de sa puissance, plaça sous sa juridiction toutes les contrées productrices d'arbres pouvant être utilisés pour la production de vaisseaux (Ojeda, 1989).

Le feu a été un autre grand facteur déterminant dans l'évolution des forêts de la péninsule pendant des millénaires - sa présence à la surface de la terre étant d'ailleurs très antérieure à l'apparition de l'homme et, très probablement, à l'apparition des formations végétales de caractère méditerranéen. Le charbon de bois fossile, produit par les incendies qui dévastèrent les forêts du Dévonien il y a 375 millions d'années, permet aux scientifiques d'affirmer que de gigantesques feux naturels détruisirent de 
vastes étendues de forêts dans ces moments originels de l'évolution de la vie sur la planète (Jones, 1991). L'utilisation du feu par les premiers groupes d'hominidés semble remonter à quelques 400000 ans B. P., selon les données archéologiques du site de Terra Amata, près de Nice, localisé dans une petite calanque, à 26 mètres d'altitude (Santacana, 1987). Les restes découverts correspondent à un campement de nomades enclavé dans un bois de conifères, dont les troncs étaient utilisés comme bois de chauffe.

Les forêts ont disparu lentement avec la révolution agricole néolithique, le surpâturage et l'utilisation du feu. Le désir de gagner des terres fut un des premiers facteurs qui incitèrent à couper des arbres et à ouvrir des clairières. De surcroît, il était assez courant de mettre le feu à la montagne : cette méthode demandait moins d'efforts, mais elle présentait l'inconvénient de ne pas permettre de contrôler facilement l'étendue de l'incendie. D'après de nombreux témoignages médiévaux (Carle, 1976), les paysans n'étaient pas les seuls à les provoquer: les bergers et les éleveurs y contribuaient largement, pour obtenir l'année suivante de meilleures pâtures.

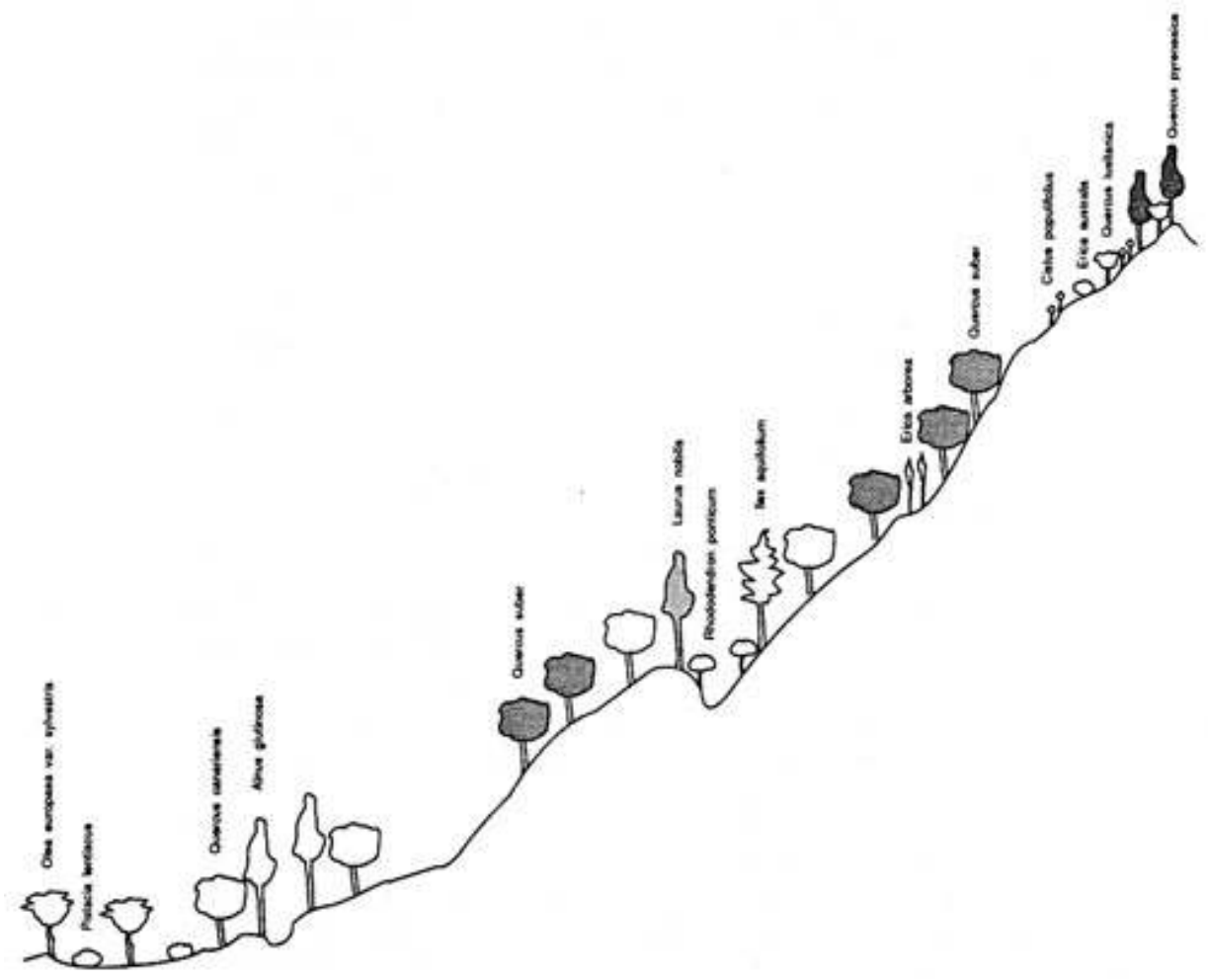

Distribution phytogéographique des espèces arborées et arbustives de la sierra de l'Aljibe (province de Cadix)

Selon toutes les estimations actuelles, l'immense majorité des incendies qui se produisent dans la région méditerranéenne sont provoqués volontairement ou involontairement par l'homme, même si les caractéristiques du climat, comprenant une longue période sèche coïncidant avec de hautes températures, favorisent notablement les conditions de départ de ces incendies. Par ailleurs la végétation est résistante à la sécheresse, avec d'abondantes structures ligneuses, xérophiles et sclérophylles, et donc avec une faible teneur en eau (Riba, 1987). De plus, des mécanismes d'adaptation pour se protéger de la répétition des incendies ont dû se développer; ils pourraient être à la base de changements ultérieurs dans la richesse 
floristique, ce qui a été bien étudié pour les garrigues françaises (Trabaud, 1980). Fréquemment, les plantes méditerranéennes sont également riches en essences volatiles et l'ensemble de la végétation produit de considérables accumulations de biomasse, c'est-à-dire un matériau facilement combustible et inflammable. Si nous ajoutons à tout cela l'ancienneté de la présence humaine, on peut certifier que le paysage méditerranéen est le résultat d'une "co-évolution" avec l'homme (Naveh, 1975). En Andalousie les incendies de forêts au cours des trois dernières décennies ont détruit plus d'un demi-million d'hectares (voir figure 1) et, comme dans tous les pays du bassin méditerranéen, cela pose un grave problème d'environnement.

Les Sierras de l'Aljibe et de la région de Gibraltar

Dans la province de Cadix, la plus méridionale d'Europe, subsistent encore de grands espaces arborés ayant un authentique caractère forestier, qui, du fait des caractéristiques des strates arborée, arbustive et lianoïde, prennent l'apparence en certains lieux de forêts très denses, probablement très semblables à celles qui ont couvert de grandes étendues de l'Europe du Sud il y a trois ou quatre millions d'années.

Dans la zone du détroit de Gibraltar s'est maintenu un ensemble forestier qui se distingue par son taux élevé de conservation et sa biodiversité. La nature particulière du substrat dominant, constitué par des grès du Miocène à caractère acide, a favorisé une flore très riche et très variée, ce qui depuis des siècles a attiré dans ces montagnes de nombreux botanistes nationaux et étrangers. Dans le seul secteur le plus méridional, c'est-à-dire dans les Sierras d'Algéciras, on a dénombré 745 espèces (Gil,1985). Les formations végétales sont des forêts de chênes-lièges (Quercus suber), et, sur une moindre étendue, de chênes des Canaries (Quercus canariensis), qui semblent adaptées aux conditions orographiques et micro-climatiques faisant de ces montagnes des lieux parmi les plus riches et les plus précieux d'un point de vue biogéographique et paysager. A côté de ces formations apparaissent des espèces à caractère lauroïde, reliques du Tertiaire, comme le rhododendron (Rhododendron ponticum sous-espèce baeticum), le laurier (Laurus nobilis), le cornouiller (Viburnum tinus), etc., qui confèrent à la zone ses caractéristiques particulières.

Ces Sierras de l'Aljibe et de la région de Gibraltar sont actuellement en grande partie protégées, depuis qu'elles ont été décrétées Parc naturel par l'Agence de l'environnement, l'organisme autonome qui veille à leur conservation et à leur exploitation raisonnée. La superficie protégée dépasse 175000 hectares, et inclut toute une série de moyennes montagnes qui culminent au Pic de l'Aljibe, à 1092 mètres. La série de formations végétales que nous étudions, dans leur structure végétale actuelle et dans leur évolution historique, représentée sur la figure 2, est très riche quant au nombre d'espèces différentes de Quercus qui y apparaissent, tant arborées qu'arbustives (Jurado, 1993).

Bien que les caractéristiques climatiques soient, à grands traits, méditerranéennes, il est certain que les fréquents brouillards dans les zones élevées et la durée et l'intensité des vents d'est dans cette région provoquent un taux persistant d'humidité qui, dans certains endroits (Llanos del Juncal) donne à l'ensemble un aspect de «forêt de brouillard». La proximité de l'océan Atlantique et de la Méditerranée contribue à adoucir les températures, dont la moyenne annuelle oscille autour de $15-17^{\circ} \mathrm{C}$ sur la ligne côtière, et diminue un peu vers l'intérieur des montagnes, ainsi qu'avec l'altitude. La pluviométrie est assez élevée, atteignant $1356 \mathrm{~mm}$ à Puerto Gáliz (417 mètres), et dépassant les $2000 \mathrm{~mm}$ aux sommets de l'Aljibe. Pendant les mois d'été, les 
précipitations sont négligeables. Mais l'existence de cours d'eau permanents et l'humidité que produit le vent d'est sur les hauteurs font que ces vallées chaudes, appelées localement canutos (roseau, tuyau NDT), sont devenues le refuge d'une flore exubérante de type subtropical, comme celle qui devait couvrir les côtes à la fin du Tertiaire et qui a dû disparaître presque complètement durant les grandes glaciations qui affectèrent l'Europe au Pléistocène.

Pour l'étude et la caractérisation des formations végétales actuelles, nous utilisons des méthodes quantitatives d'estimation de la couverture, de la densité arborée et d'autres paramètres qui nous permettent une caractérisation précise de l'écosystème. Les mesures se font depuis plus de deux ans, réparties de manière sélective dans l'ensemble du Parc, en définissant une série de zones de plus grande richesse et valeur forestière. On a réalisé à ce jour cinquante transects et, dans chacun d'eux, avec un ruban métrique de cent mètres de long, on a déterminé la densité arborée, en utilisant la méthode des « couples au hasard » (Cottan, 1955), déjà utilisée par les agronomes nordaméricains. Elle est fondée, on le sait, sur l'utilisation d'un angle d'exclusion pour déterminer une série de paires d'espèces. La distance moyenne obtenue est multipliée par un coefficient de correction et permet d'estimer la densité d'arbres par hectare dans cette zone. Cette densité peut conserver un rapport avec les actions humaines antérieures, et l'estimation du diamètre des arbres, mesurés à 1,3 mètre du sol, nous permet d'apprécier l'âge de la forêt avec un certain degré de précision. De plus chaque transect est défini par une série de données relatives à la présence ou l'absence d'espèces, aux incendies, etc., et s'accompagne également d'autres paramètres tels que l'altitude, l'orientation ou la pente.

La caractérisation de ces imposantes forêts de Quercus du sud de l'Europe et l'étude du degré d'intervention de l'homme au cours des derniers siècles nous permettront de mieux connaître les écosystèmes méditerranéens et leur capacité de réponse face aux agents externes et, dès lors, d'améliorer leur gestion.

\section{BIBLIOGRAPHIE}

BAUER MANDERSCHEID, E. (1980), Los Montes de España en la Historia, Madrid, Ministerio de Agricultura y Pesca.

BLANCA, G. (1993), « Origen de la Flora Andaluza », in VALDES B., Introducción a la Flora Andaluza, Séville, Junta de Andalucía.

CARLE, M.C. (1976), «El Bosque en la Edad Media », Buenos Aires, Cuadernos de Historia de España, LIX, LX.

CASTROVIEJO BOLIBAR, S., GARCIA DORY, M.A., MARTINEZ VICENTE, S., \& PRIETO, F. (1985), «Política Forestal en España », Madrid, Quercus, 19.

COSTA TENORIO, M., GARCIA ANTON, M., MORLA JUARISTI, C. \& SAINZ OLLERO, H. (1990), « La evolución de los bosques de la Península Ibérica : una interpretación basada en datos paleobiogeográficos », Madrid, Ecologia, Hors-série 1, ICONA. 
COTTAN, G. \& CURTIS, J.T. (1955), « Correction for various exclusion angles in the randon pairs methods ", Ecology, 37, pp. 451-460.

GIL, J.M., ARROYO, J. \& DEVESA, J.A. (1985), « Contribución al conocimiento florístico de las Sierras de Algeciras (Cádiz, España) », Acta Botánica Malacitana, 10, pp. 97-122.

GONZALEZ BERNALDEZ, F. (1989), « Influencia humana en los ecosistemas forestales », Madrid, Quercus, 37.

GRANADOS CORONA, M. (1987), Transformaciones históricas de los ecosistemas del Parque Nacional de Doñana, Thèse de Doctorat, Université de Séville (inédite).

GROOME, H. (1990), Historia de la política forestal en el Estado español, Madrid, Agencia de Medio Ambiente.

HERNANDEZ DEL AGUILA, R. (1985), La crisis ecológica, Barcelone, Laia.

JONES, T.P. \& CHALONER, N.S. (1991), « Los incendios del pasado », Barcelone, Mundo Científico, 119, vol. 11.

JUNTA DE ANDALUCIA (1989), Plan Forestal Andaluz, Séville, Consejería de Agricultura y Pesca. JURADO DOÑA, V. \& BORRERO BORRERO, M.L. (1992), La Dehesa, Monografía n² 2, Plan Forestal Andaluz, Séville, Consejería de Agricultura y Pesca.

JURADO DOÑA ,V. \& OJEDA RIVERA, J.F. (1993), « Ecología de los bosques de Quercus del Parque Natural de Los Alcornocales », Pontevedra, Actas del Congreso Forestal Español, tome I, pp. 387-390.

KLEIN, J. (1979), La Mesta, Madrid, Alianza Editorial (première édition en 1940).

MARAÑON ARANA, T. (1988), « Agro-sylvo pastoral systems in the Iberian Peninsula : Dehesas and Montados ", Rangelands, 10, 6, pp. 255-258.

NAVEH, Z. (1975), « The evolutionary significance of Fire in the Mediterranean Region », Vegetatio, vol. 29, 3, pp. 199-208.

OJEDA RIVERA, J.F. (1989), « El Bosque andaluz y su gestión a través de la historia », in CANO GARCIA G. (dir.), Geografía de Andalucía., Edit. Tartessos, tome V, pp. 315-355.

PARRA F., 1988, La Dehesa y el Olivar, Barcelone, Debate/Circulo.

PONS, A. \& QUEZEL, P. (1985), « The history of the flora and vegetation and past and present human disturbance in the Mediterranean Region ", in GOMEZ CAMPO C. (Ed.), Plant Conservation in the Mediterranean area, La Haye, Dr. Junk.

PEREZ, C. \& TERES, J. (1991), « La red de vías pecuarias en España », Madrid, Quercus, 68.

RIBA, M. \& TERRADAS, J. (1987), « Característiques de la resposta als incendis en els ecosistemes mediterranis », Barcelone, Quaderns d'ecologia aplicada, 10, Diputació de Barcelona.

SAENZ RIDRUEJO, C. et al. (1986), « Las rutas de la Mesta », Los Cuadernos de Cauce 2000, 10, Madrid, Colegio de Ingenieros de Caminos, Canales y Puertos.

SANTACANA, J. (1987), Las Primeras sociedades, Madrid, Editorial Anaya, Biblioteca Básica de Historia.

STEVENSON, A.C. \& HARRISON, R.J. (1992), « Ancient forests in Spain : A model for land-use and dry forest management in South-west Spain from $4000 \mathrm{BC}$ to $1900 \mathrm{AD} »$, Proceedings of the Prehistoric Society, 58, pp. 227-247.

SUC, J.P. (1985), «El clima mediterráneo : ¿ Una particularidad de siempre? », Barcelone, Mundo Científico, 51. 
THIRGOOD, J.V. (1981), Man and the Mediterranean Forest. A history of resource depletion, Londres, Acad. Press.

TRABAUD, L. (1980), Impact biologique et écologique des feux de végétation sur l'organisation, la structure et l'évolution de la végétation des zones de garrigues du Bas-Languedoc, Thèse d'Etat, Montpellier, Univ. Sc. Tech. du Languedoc.

VERNET, J.L. (1973), « Etude sur l'histoire de la végétation du sud-est de la France au Quaternaire, d'après les charbons de bois principalement », Paléobiol. Continent., 4(1), pp. 1-90.

\section{NOTES}

1. Le mot matorral désigne des formations arbustives de tout type, sans dépendance édaphique, et sans relation avec le caractère plus ou moins fermé de la formation ; on ne peut donc pas assimiler cette formation au maquis (NDLR).

2. Comme le précisent Josefina Gómez Mendoza et al. dans ce numéro, le terme dehesa fait référence à un domaine foncier clôturé, avec une couverture d'arbres plus ou moins dense, qui donne lieu à différentes formes d'exploitation : exploitation forestière (charbon de bois, bois d'oeuvre, autres produits), élevage (pâturages), agriculture (culture céréalière) (NDLR).

3. Société d'encouragement et de surveillance pour l'élevage.

\section{RÉSUMÉS}

Comme d'autres forêts méditerranéennes, les forêts d'Andalousie ont été modifiées au cours des derniers millénaires par l'activité humaine, notamment du fait des coupes et incendies. L'auteur fait ressortir le rôle de la Mesta, une institution qui s'est maintenue en Espagne pendant des siècles. Dans un second temps il décrit la variété écologique et floristique des espaces arborés qui se sont maintenus dans la zone du détroit de Gibraltar, et présentent un authentique caractère forestier.

\section{Biogeography of a mediterranean forest space.}

As other Mediterranean forests, Andalousian forests have been modified, since very ancient times, by human activities (cuttings, fires...), in which the centuries old institution of the Mesta has played a great part. In the biogeographic area of the Gibraltar Sierra, forest spaces deserve to be described in order to underline the ecological and floristical variety of arborescent species, jointly with historical structuring aspects of forestry.

\section{Biogeografia de un espacio forestal mediterráneo español.}

Se constata la actual importancia de los bosques de Andalucía que, al igual que otros bosques mediterráneos, han sido modificados en los últimos milenios por la acción humana (talas, fuegos), destacándose el papel de la Mesta, una institución de carácter ganadero que ha perdurado en España durante siglos. Se destaca asimismo la variedad ecológica y florística de la región biogeográfica de las Sierras del Aljibe y del Campo de Gibraltar y la acción histórica sobre la estructura de esos espacios forestales. 
INDEX

Mots-clés : Espagne, Andalousie, écosystème, biogéographie, Parcs naturels, Fonctionnement de l'écosystème, Pratiques agraires et pastorales, Dehesa, Incendies forestiers, Parc des Alcornocales Palabras claves : España, Andalucia, biogeografía, Parques naturales, Funcionamiento del ecosistema, Prácticas agrarias y pastorales, Incendios forestales, Parque de los Alcornocales Keywords : Spain, Andalousia, biogeography, Natural parks, Ecosystem, Agricultural and rural practices, Forest fires, Alcornocales Park

\section{AUTEUR}

\section{VICENTE JURADO DOÑA}

Biologiste, il est actuellement directeur-conservateur du Paraje natural de « Marismas del Odiel » (Huelva). Sa thèse de doctorat porte sur la biogéographie et les transformations historiques des forêts de chênes du Parc naturel des Alcornocales (Cadix). 